\title{
TIME DEVELOPMENT OF MULTIPLE POLARIZATION ZONES IN PVDF
}

\author{
G. Eberle, B. Dehlen, W. Eisenmenger, \\ 1. Physikalisches Institut, University of Stuttgart, Germany
}

\begin{abstract}
The polarization distribution in PVDF which causes the piezo- and pyroelectricity of this polymer depends strongly on the polarization distribution. Applying an electric field of $E=60 \mathrm{MV} / \mathrm{m}$ causes a ca. $10 \mu \mathrm{m}$ thick polarization zone in the middle of the sample. Applying the same field in opposite direction a bimorph structure results. If the same field is applied to a homogeneously prepolarized PVDF sample a trimorph structure is formed with three polarization zones under field. But under short circuit conditions the polarization zone in the new field direction vanishes. The polarization distribution is measured with the acoustic PPS method with a spatial resolution of about $2 \mu \mathrm{m}$.
\end{abstract}

\section{Introduction}

Since the discovery of the piezo- and pyroelectricity of PVDF $[1,2]$ this semicrystalline and ferroelectric polymer is of great interest. Because of its easy handling it is used in many technical applications for example as hydrophone, microphone, infrared detector, etc.. In addition the acoustic impedance of PVDF is of the same order of the acoustic impedance of water and the most organic materials. The piezoelectric coefficients of PVDF are comparabel to these of quartz.

To use PVDF films for piezoelectrical applications the films have to be poled in electric fields of about $100 \mathrm{MV} / \mathrm{m}$. In this strong fields the molecular dipoles orient in field direction by chain rotation in steps of $60^{\circ}$ within the crystallites [3]. After switching off the field the polarization in the crystallites is remanent. This remanent polarization causes the piezo- and pyroelectricity of PVDF.
Simultaneously to the dipole orientation charges are injected from the electrodes during the poling process [4]. Experiments with blocking electrodes [5] and the fact that the time development of the remanent polarization is delayed by a factor $3 . . .5$ compared to the dipol alignment under field $[6,7]$ indicate that the polarization is stabilized by Coulomb interaction of deeply trapped charges at the crystallite surfaces. These charges are ions which are created by electrochemical reactions at the electrodes [8].

For technical applications in sensors and other activators it is of great interest that the polarization distribution within thickness direction of the PVDF films depends strongly on the poling condition. By constant voltage poling only small, ca. $10 \mu \mathrm{m}$ thick polarization zones are observed at low fields $(\sim 50 \mathrm{MV} / \mathrm{m})$ while the rest of the films is free of polarization. At high fields $(\sim 200 \mathrm{MV} / \mathrm{m})$ the polarization is homogeniously distributed in thickness direction [4]. Besides this the polarization distribution is also influenced by the proportion of non polar $\alpha$ and polar $\beta$ phase crystallites [9]. To demonstrate the importance of the poling conditions we present the time development of two and three (bimorph and trimorph), $10 \ldots 15 \mu \mathrm{m}$ thick polarization zones with different signs.

\section{Experimental}

The polarization distribution in thickness direction of the PVDF films was measured with the PPS methode (Piezoelectrically induced Pressure Step) $[10]$ as illustated in fig. 1 . With the help of a reed switch a coaxial cable is discharged. This produces a voltage step u of $600 \mathrm{~V}$. The voltage step is transformed in a pressure step by a piezoelectric X-cut quartz. The rise time of the pressure step is about 


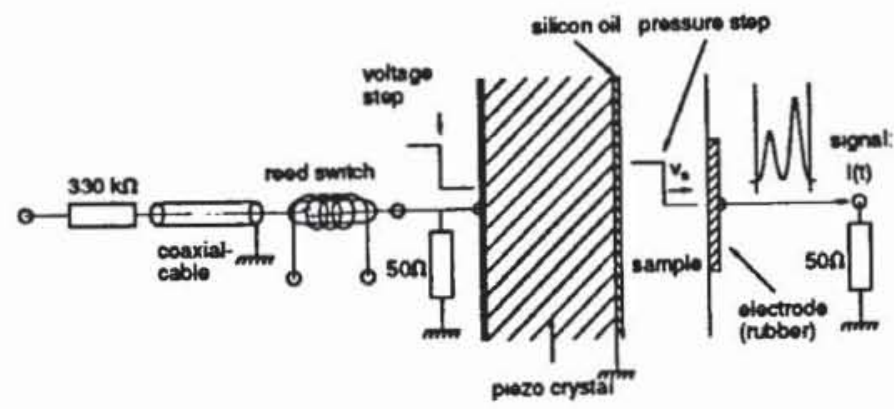

Figure 1: Block diagramm of the PPS apparatus.

1ns. It is coupled to the PVDF samples and propagates with sound velocity. Real and polarization charges are displaced and deformed by this propagating pressure step. The corresponding time dependent displacement current $I(t)$ is is measured with a broad band amplifier and displayed on an $1 \mathrm{GHz}$ real time oscilloscope screen. The signal is camera recorded on a video tape and can be digitized afterwards. In this way we obtain the time dependence of the charge and polarization development with a time resolution of $20 \mathrm{~ms}$ for many hours. The spatial resolution depends on the rise time of the pressure step and on the sound velocity and is about $2 \mu \mathrm{m}$ for PVDF. The polarization distribution can be directly calculated from the displacement current $\mathrm{I}(\mathrm{t})[10]$ :

$$
I(t)=E \cdot K \cdot C \cdot D\left(x=v_{s} t\right)=\left.E K C \cdot P(x)\right|_{E=0}
$$

E... excitation constant: $E=v_{s}^{\prime} \cdot d^{\prime} \cdot u / l^{\prime}$

$$
\text { K... coupling faktor: } \quad K=2 Z^{\prime} /\left(Z+Z^{\prime}\right)
$$$$
\text { C... sample capacity: } \quad C=A / l
$$

$\left(v_{s}^{\prime}, d^{\prime}, l^{\prime} \ldots\right.$ sound velocity, piezoelectric constant and thickness of X-cut quartz respectively; $u$... voltage pulse height; $Z, Z^{\prime}$... acoustic impedances of PVDF and quartz; $A, l \ldots$ sample area and thickness of the PVDF sample).

A thin, $25 \mu \mathrm{m}$ thick Al-evaporated and high isolating PET film is inserted between the PVDF sample and measuring electrode. With its help high voltage up to $8 \mathrm{kV}$ can be applied to the sample during the PPS analysis. The PET doesn't change the shape of the signal because it acts as a wide band coupling capacitor.

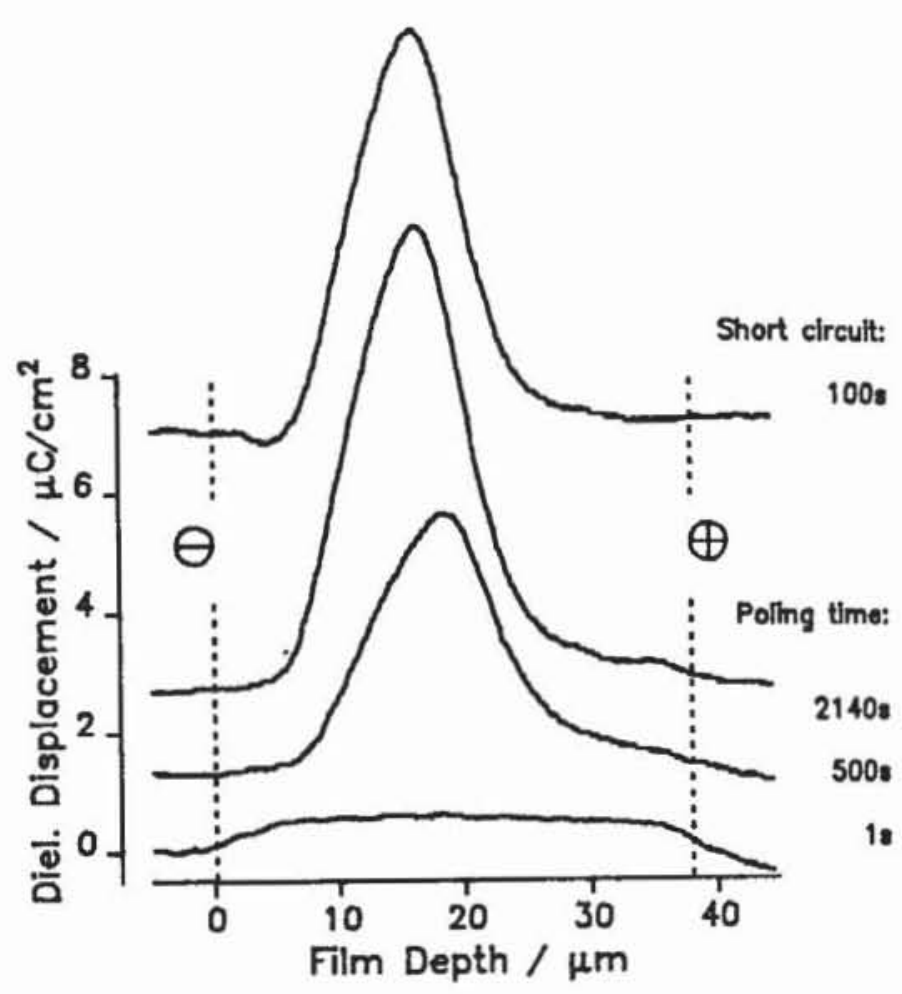

Figure 2: Formation of a ca. $10 \mu \mathrm{m}$ thick polarization zone in the middle of a $30 \mu$ thick PVDF film. Illustrated is the dielectric displacement under field $(E=60 M V / m)$ at different poling times and the remanent polarization after 100 s under short circuit conditions.

\section{Results}

\subsection{Bimorph-Structur}

Figure 2 shows the time dependent formation af a $15 \mu \mathrm{m}$ thick polarisation zone in the middle of a $38 \mu \mathrm{m}$ thick biaxially stretched PVDF film with about $70 \% \beta$ content at $\mathrm{E}=60 \mathrm{MV} / \mathrm{m}$. After $1 \mathrm{~s}$ poling time an almost homogenious distribution of the dielectric displacement is seen with charges near the electrodes. After 500s homo charges are injected from the electrodes in a ca. $10 \mu \mathrm{m}$ thick zones near both electrodes which are now field free. Therefore the electric field is increased in the middle of the sample. In this increased field the crystallite dipoles are oriented and the displacement increases between 10 and $25 \mu \mathrm{m}$ film depth. At $2140 \mathrm{~s}$ poling time the displacement in the middle of the sample is increased to $7.7 \mu \mathrm{C} / \mathrm{cm}^{2}$. Afterwards the 


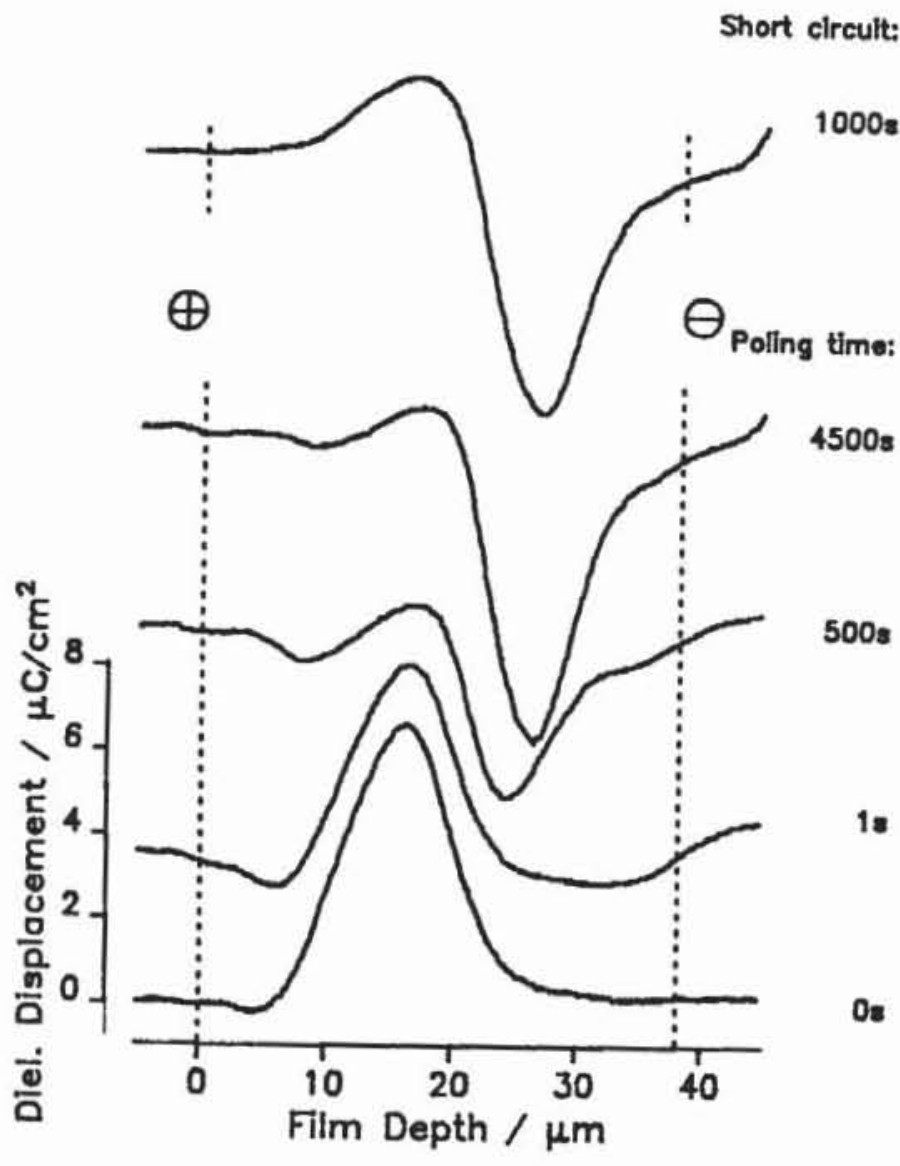

Figure 3: Formation of a second ca. 10 $\mu \mathrm{m}$ thick polarization zone with opposite sign after a field of $E=-60 M V / m$ has been applied to the sample prepolarized as described in figure 2.

sample is kept under short circuit conditions. After the dielectric relaxation time of about 100 s the free charges vanish in PVDF and remanent polarization of $6.5 \mu \mathrm{C} / \mathrm{cm}^{2}$ remains between 10 and $20 \mu \mathrm{m}$ film depth.

Now an electric field of $\mathrm{E}=60 \mathrm{MV} / \mathrm{m}$ is applied in opposite direction to the same sample. This is illustrated in figure 3 . The polarization distribution after 0 s poling time in figure 3 is the same as in figure 2 after 100 s under short circuit conditions. After 1s under field in opposite direction the polarization in the middle of the sample is reduced and a small displacement in negative direction is seen in the $10 \mu \mathrm{m}$ thick boundary zones. By further poling the first polarization zone in the middle decreases within the first 500 s. But directly on the right side of the polarization zone in figure 3 a second $10 \mu \mathrm{m}$ polarization zone increases with opposite sign until 4500 s poling time. Then the sample is kept under short circuit conditions for a second time. After 1000 s under short circuit conditions the first polarization zone in the middle of the sample rebuilts from $0.5 \mu \mathrm{C} / \mathrm{cm}^{2}$ to $2 \mu \mathrm{C} / \mathrm{cm}^{2}$ whereas on the right side a second polarization zone with $6 \mu \mathrm{C} / \mathrm{cm}^{2}$ in the opposite direction is remanent. With this poling procedure we have produced a bimorph structure of the polarization. This bimorph structure consists of $2 \mathrm{ca}$. $10 \mu \mathrm{m}$ thick polarization zones side by side with opposite sign in the middle of a $38 \mu \mathrm{m}$ thick PVDF film. The boundaries of the film are free of polarization.

\subsection{Trimorph-Structure}

In figure 4 an electric field of $60 \mathrm{MV} / \mathrm{m}$ is applied to a $38 \mu \mathrm{m}$ thick PVDF sample which has been poled for $330 \mathrm{~s}$ at $180 \mathrm{MV} / \mathrm{m}$ in opposite direction. After this prepolarization process the remanent polarization was almoust homogeneously distributed in thickness direction with a value of $5.5 \mu \mathrm{C} / \mathrm{cm}^{2}$ (not shown in figure 4). Applying the electric field in the new, opposite direction (see figure 4) the polarization is reduced to $2.5 \mu \mathrm{C} / \mathrm{cm}^{2}$ at $10 \mathrm{~s}$ poling time. By further poling the polarization in ca. $10 \mu \mathrm{m}$ zones at the sample boundaries is slowly reduced to $1.5 \mu \mathrm{C} / \mathrm{cm}^{2}$. This polarization remains constant by further poling. But in the middle of the sample a new, ca. $15 \mu \mathrm{C} / \mathrm{cm}^{2}$ thick polarization zone grows in field direction with opposite sign compared to the polarization at the boundaries. In figure 4 this polarization zone in the middle of the sample is seen as a reduction of the polarization to $0.5 \mu \mathrm{C} / \mathrm{cm}^{2}$ and the creation of an inhomogenious spatial distribution. At 19990s a trimorph results with about $1.5 \mu \mathrm{C} / \mathrm{cm}^{2}$ polarization at the boundaries in the original direction and also $1.5 \mu \mathrm{C} / \mathrm{cm}^{2}$ in a central polarization zone in field direction. The polarization at the boundaries cannot be inverted so easy compared to the polarization in the middle of the film.

Under short circuit conditions the polarization in the central zone is reduced to $0 \mu \mathrm{C} / \mathrm{cm}^{2}$ whereas the 


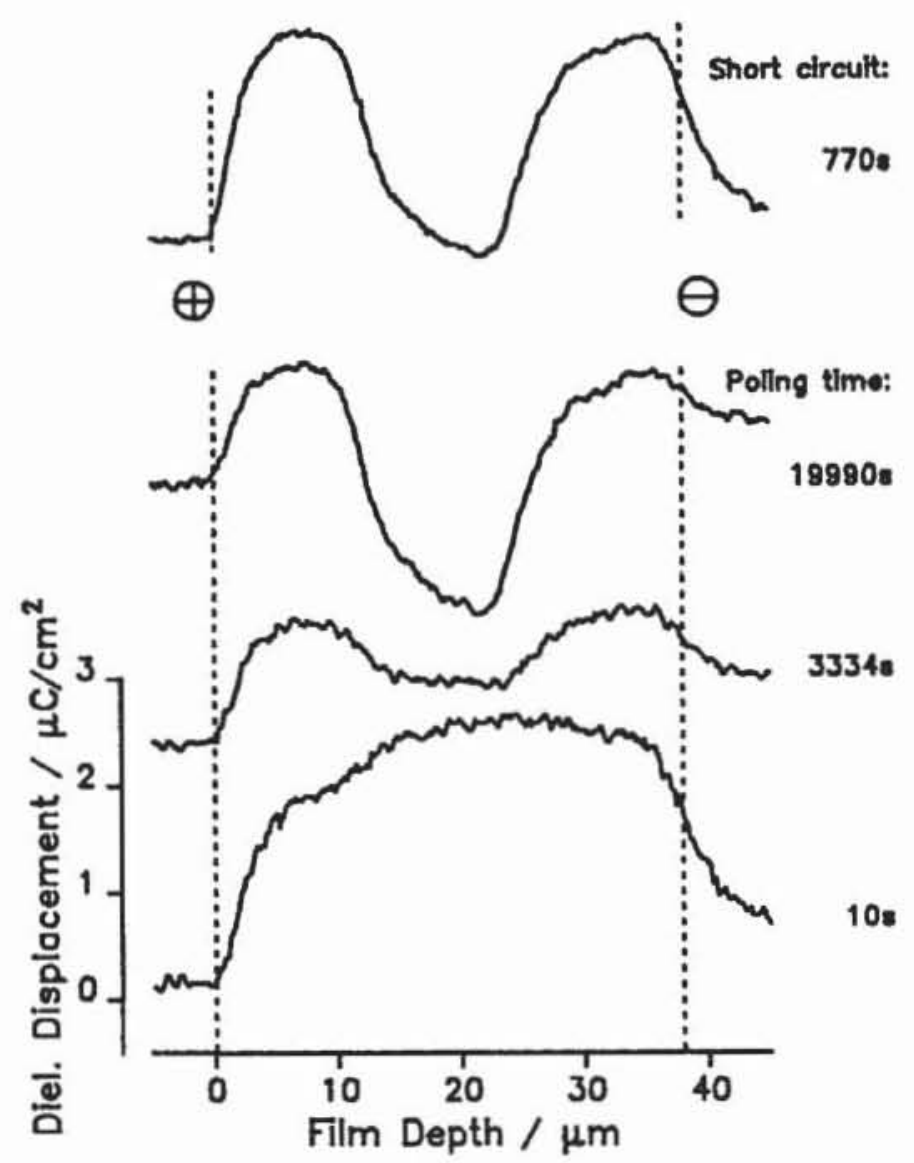

Figure 4: Formation of a trimorph structure after applying of an electric field of $E=60 \mathrm{MV} / \mathrm{m}$ to a $38 \mu m$ thick PVDF sample After prepoling 330 s at $E=180 \mathrm{MV} / \mathrm{m}$ the sample had an almost homogeneous distributed remanent polarization of $5.5 \mu \mathrm{C} / \mathrm{cm}^{2}$.

polarization zones at the boundaries are increasing to $2 \mu \mathrm{C} / \mathrm{cm}^{2}$.

\section{Summary}

In this paper we have shown two examples with the help of the acoustic PPS apparatus that the spatial polarization distribution in thickness direction in PVDF which is responsibel for the piezoelectricity of this polymer strongly depends on the poling conditions. In the first example we poled a $38 \mu \mathrm{m}$ thick PVDF film with $\mathrm{E}=60 \mathrm{MV} / \mathrm{m}$. The result was a small, ca. $10 \mu \mathrm{m}$ thick polarizaton zone in the middle of the film. After applying the same field in opposite direction a bimorph structure was built with two ca. $10 \mu \mathrm{m}$ thick polarization zones side by side. In the second example an electric field of $\mathrm{E}=60 \mathrm{MV} / \mathrm{m}$ was applied 20000 s to a sample with a homogeneous polarization distribution after prepolarization. Under field a trimorph structure was created with two polarization zones in the original direction at the film surfaces and an polarization zone in opposite direction in the middle of the sample. Under short circuit conditions the polarizations at the surfaces was remanent wheras the polarization in the middle vanished.

\section{References}

[1] H. Kawai, Jap. J. Phys., 8, p. 975, 1969

[2] Jr. J. G. Bergmann, J. H. McFee, G. R. Crane, Appl. Phys. Lett., 18, p. 203, 1971

[3] R. G. Keppler, R. A. Anderson, J. Appl. Phys., 49, p.1232, 1978

[4] K. Holdik, W. Eisenmenger, Proc. 5th Intern. Sym. Electrets, Piscataway, p.553, 1985

[5] E. Bihler, G. Neumann, G. Eberle, W. Eisenmenger,

IEEE Annual Report Conf. Elect. Insul. and Diel. Phen., Piscataway, p. 140, 1990

[6] M. Womes, E. Bihler, W. Eisenmenger, IEEE Trans. Electr. Insul., 24, p. 461, 1989

[7] G. Eberle, E. Bihler, W. Eisenmenger, IEEE Trans. Electr. Insul., 26, p.62, 1991

[8] M. Selle, G. Eberle, E. Bihler, W. Eisenmenger,

IEEE Annual Report Conf. Elect. Insul. and Diel. Phen., Piscataway, p. 87, 1992

[9] E. Bihler, K. Holdik, W. Eisenmenger, IEEE Trans. Electr. Insul., 24, p. 541, 1989

[10] W. Eisenmenger, M. Haardt, Solid State Comm., 41, p.917, 1982 